\title{
Desarrollo del sistema motriz virtual de una plataforma subacuática móvil inspirada en biomimetismo
}

\author{
Development of a virtual drive system of an underwater mobile \\ platform inspired in biomimicry
}

\author{
Rubén Darío Muelas H. ${ }^{1} \quad$ José Tomás Buitrago $^{1} \quad$ Breyner Posso Bautista $^{1}$ \\ Recibido 5 de abril de 2013, aceptado 5 de marzo de 2014 \\ Received: April 5, $2013 \quad$ Accepted: March 5, 2014
}

\begin{abstract}
RESUMEN
En este artículo se presenta el modelo virtual de una plataforma subacuática móvil inspirada en biomimetismo seleccionando como sistema de locomoción el modo de un pez subcarangiforme, el que se basa en las aletas caudal y de cuerpo (BCF Body and Caudal Fins) para su propulsión. Este modo utiliza las dos terceras partes del cuerpo para generar el empuje y se caracteriza por ser un modo de alta maniobrabilidad. Para el desarrollo del modelo se llevó a cabo un modelamiento del prototipo en 3D utilizando un software de diseño asistido por computador (CAD), un análisis considerando la cinemática directa e inversa y un sistema de control para la navegación implementando la lógica difusa. En este modelo se desarrolló un algoritmo basado en métodos geométricos para dar solución a la cinemática inversa considerando el área de trabajo de la plataforma y dividiéndola por subáreas y así poder llevar a cabo un análisis de posición, velocidad, aceleración y torque de cada articulación del pez robótico. También se desarrolló un algoritmo de navegación utilizando la lógica difusa como método de control no lineal. Los resultados obtenidos representan una aproximación del comportamiento de los peces modo subcarangiforme que son de gran interés para el futuro desarrollo e implementación de una plataforma subacuática física inspirada en biomimetismo.
\end{abstract}

Palabras clave: BCF, biomimetismo, CAD, cinemática, lógica difusa, modelamiento matemático, pez robótico, subcarangiforme.

\section{ABSTRACT}

This paper presents the model of a virtual drive system of an underwater mobile platform inspired by biomimicry, selecting the subcarangiform mode of fishes as locomotion system which is based on body and caudal fins $(B C F)$. This model uses two-thirds of the body for thrust and one of the most important features is the high maneuverability. The development of the model is based on simulation by designing the $3 D$ model of the prototype using a computer aided design software (CAD), an analysis by considering the direct and inverse kinematics and a navigation control system by implementing the fuzzy logic. The algorithm implemented to solve the inverse kinematics is based on geometric methods considering the workspace and dividing it into subareas in order to analyze position, velocity, acceleration and torque for every joint of the robot fish. Also, a navigation control algorithm was developed using the fuzzy logic as non-linear control method. The results represent an approximation of subacarangiform mode of fish behaviours for future physical implementation of an underwater platform inspired by biomimicry.

Keywords: BCF, biomimicry, CAD, fuzzy logic, kinematics, mathematical model, robot fish, subcarangiform.

\footnotetext{
1 Escuela de Ingeniería Eléctrica y Electrónica. Universidad del Valle. Calle13 № 100-00. Cali, Colombia. E-mail: rubendmh4@hotmail.com; jose.buitrago@correounivalle.edu.co; breyner.posso@correounivalle.edu.co
} 


\section{INTRODUCCIÓN}

El desarrollo e implementación actual de cualquier plataforma subacuática es un proceso costoso que incluye un estudio detallado del comportamiento y propiedades físicas del cuerpo y el medio. Cualquier desarrollo en esta área implicaría en el entorno la construcción o compra del hardware que lo soporte. Esta situación conlleva a que los procesos de aprendizaje e investigación se vean retardados, limitando así la posibilidad de desarrollar nuevos métodos y sistemas. Buscando una solución a este problema, este artículo plantea la utilización de nuevas técnicas en este campo que permitan simular plataformas subacuáticas sin necesidad de ser fabricadas, lo que reduciría considerablemente la fase de experimentación y generaría una disminución en los costos, un aumento de la productividad y la calidad, haciéndolas así más competitivas.

El biomimetismo es el arte de usar la inspiración biológica en la ingeniería para simular y emular el desempeño de plataformas que se comportan como seres vivos [1]. La implementación de una plataforma subacuática para la investigación de nuevas fuentes de energía, alimento y minerales es un procedimiento poco común y costoso debido a su alto grado de complejidad y a la carencia de recursos para su desarrollo. Sin embargo, este proyecto está enfocado al desarrollo de una plataforma móvil subacuática inspirada en biomimetismo a nivel de simulación que considere los factores físicos de la plataforma y el medio, con el fin de dar un punto de partida a un proceso de investigación que permita desarrollar e implementar este tipo de plataformas a nivel físico.

Por medio de la simulación se obtuvieron datos numéricos de los efectos físicos a los que será sometida la plataforma diseñada, utilizando como herramienta el diseño asistido por computador (CAD), algoritmos para resolver la cinemática, dinámica y los algoritmos de control para la trayectoria de la plataforma. Para el desarrollo de esta plataforma se realizó un estudio detallado de cada uno de los modos de nado de los peces de las plataformas hasta ahora desarrolladas [2, 4, 8-9, 19, 24 ,27], para determinar el diseño más apropiado a desarrollar virtualmente. Este artículo será dividido en 4 partes. La primer parte corresponde a una recopilación de los modos de nado de los peces y sus movimientos asociados. La segunda parte hará énfasis en la cinemática directa e inversa desarrollada para el modo de nado seleccionado, la tercer parte presenta el algoritmo de control implementado, y por último, se presentan los resultados obtenidos.

\section{CLASIFICACIÓN DE LOS SISTEMAS DE LOCOMOCIÓN DE LOS PECES}

La clasificación de la locomoción de los peces se basa en dos factores principales [3, 12, 25]: el primer factor consiste en la forma de movimiento del cuerpo y el segundo en la estructura o segmentos de las aletas. El primer factor consiste en dos tipos de movimientos: movimiento ondulatorio y movimiento oscilatorio; y el segundo factor es el que contribuye en mayor parte a la generación de la propulsión [27]. Con estas dos clasificaciones, se define que los peces nadan utilizando dos tipos diferentes de movimiento [14], uno de ellos es con el uso de aletas pares y/o impares (MPF-Median and/or paired fin), que consiste en el movimiento de las aletas pectoral y pelviana $[6,12]$, y el otro es utilizando el cuerpo y las aletas caudales (BCF-Body and/or Caudal Fin) correspondientes a las aletas dorsal, anal y caudal. En la Figura 1 se presentan los diferentes tipos de aletas que pueden ser utilizadas por un pez.

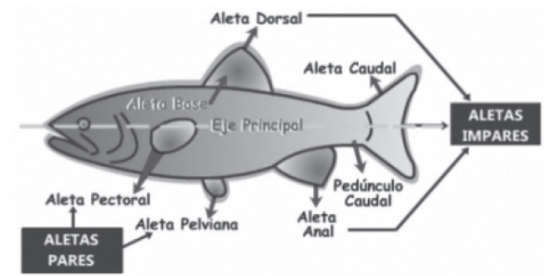

Figura 1. Clases de aletas empleadas en el movimiento de un pez

Ambos tipos de propulsión pueden ser de tipo ondulatorio u oscilatorio [2]. Los movimientos ondulatorios implican el paso de una onda a lo largo de la estructura de propulsión, es decir, el movimiento se realiza con todo el cuerpo desde la cabeza hasta la cola, mientras que en los movimientos oscilatorios la estructura de propulsión gira en un punto fijo o base sin la necesidad de formar una onda, donde el sistema de locomoción se centra principalmente en la cola. Estos dos tipos de movimientos deben considerarse simultáneamente [4], ya que los movimientos oscilatorios pueden eventualmente 
derivarse del incremento gradual de la longitud de onda en la ondulación.

Independientemente del tipo de movimiento utilizado, BCF o MPF, se pueden encontrar también varios tipos de nados frecuentemente conocidos como modos. Estos modos son identificados de acuerdo con una clasificación realizada por Breder [25], en la que los peces pueden presentar más de un modo de nado, ya sea al mismo tiempo o a velocidades diferentes debido a que muchos peces utilizan la propulsión basada en MPF para búsquedas por su alta maniobrabilidad, y para altas velocidades y altas tasas de aceleraciones cambian a propulsión BCF [13]. En la Figura 2 se presenta el diagrama de bloques de los mecanismos de propulsión.

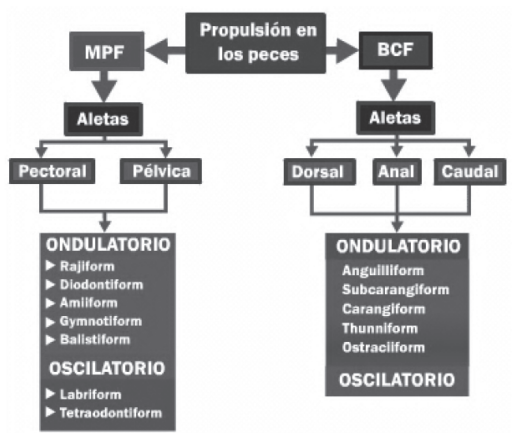

Figura 2. Diagrama de los mecanismos de propulsión en los peces [3].

A pesar de que los mecanismos MPF usan sus aletas pares e impares para generar movimientos transitorios y/o nados periódicos, los BCF además de los nados periódicos también permiten una gran maniobrabilidad, y debido a su forma hidrodinámica el análisis cinemático es menos complejo que los MPF $[5,7]$. En la Figura 3 se presentan los modos

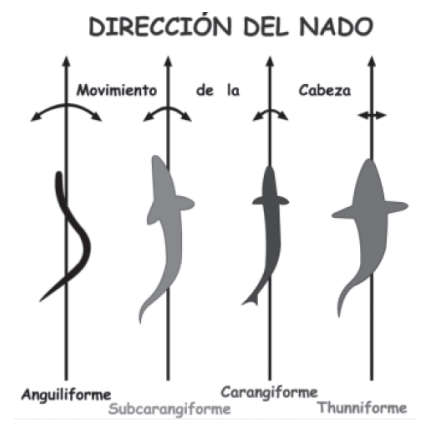

Figura 3. Dirección de nado de los mecanismos de propulsión en los peces [25]. de nado correspondientes a los BCF con sus direcciones de nado, en donde el modo anguiliforme es el que presenta un movimiento completamente ondulatorio [13] del cuerpo y el modo thunniforme un movimiento considerado como completamente oscilatorio [20]. De aquí que el ladeo sea mucho mayor en el modo anguiliforme. Los modos carangiforme y subcarangiforme presentan nados tanto ondulatorios como oscilatorios [15, 17].

Con el fin de hacer una compensación entre estos dos tipos de movimientos, entre otras características [11, $18,22]$, el modo subcarangiforme fue seleccionado para ser implementado virtualmente debido a su alta maniobrabilidad con solo utilizar las dos terceras partes de su cuerpo para la propulsión [3], y por ser un modo que permite ser configurado para actuar en los modos anguiliforme y thunniforme. Para ello se seleccionó cinco grados de libertad, como se presenta en la Figura 4.

\section{CINEMÁTICA DIRECTA E INVERSA}

Se consideraron cinco grados de libertad con el fin de que el diseño sirva de base para futuros modelos de los modos anguiliforme y thunniforme. En la Figura 5 se presentan los diferentes grados de libertad del modo seleccionado.

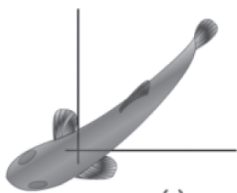

(a)

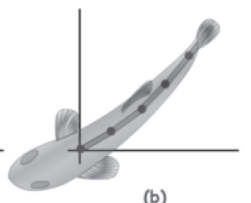

(b)

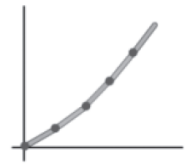

(c)
Figura 4. Grados de libertad del pez robótico, a) selección del modo, b) selección de los grados de libertad, c) representación de cuerpo del pez robótico.

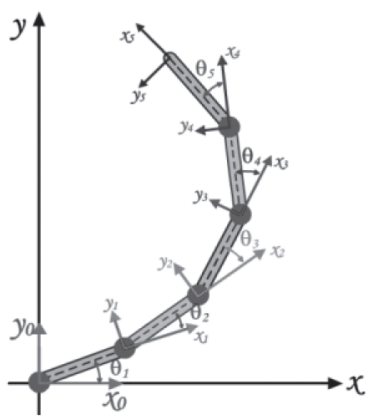

Figura 5. Representación de la cinemática directa para el cuerpo del pez modo subcarangiforme. 
Debido que el modo subcarangiforme utiliza solo las tres cuartas partes del cuerpo para su propulsión, tanto en la cinemática directa como inversa, se consideró que la cabeza del pez robótico estaba fija, aplicando así el análisis cinemático solo al cuerpo. Para solucionar la cinemática directa se seleccionó el algoritmo de Denavit Hartenberg por ser aplicable a cadenas cinemáticas de $n$-grados de libertad [30, 21], y así poder hallar la matriz homogénea total del sistema, como se presenta en la ecuación (1).

$$
T=\left|\begin{array}{llll}
n_{x} & o_{x} & a_{x} & p_{x} \\
n_{y} & o_{y} & a_{y} & p_{y} \\
n_{z} & o_{z} & a_{z} & p_{z} \\
0 & 0 & 0 & 1
\end{array}\right|
$$

Por ser un robot planar, todos los grados de libertad giran en torno al plano xy $[17,30]$, por lo tanto para la matriz homogénea total de transformación del sistema los valores $n_{z}, o_{z}, a_{z}$ y $p_{z}$ son nulos, y el resto de variables están expresadas en función de sumas de senos y cosenos de los ángulos $\theta_{1}, \theta_{2}$, $\theta_{3}, \theta_{4}$, y $\theta_{5}$, como se presenta a continuación en la ecuación (2), hasta la ecuación (10).

$$
\begin{gathered}
n_{x}=\cos \left(\theta_{1}+\theta_{2}+\theta_{3}+\theta_{4}+\theta_{5}\right) \\
o_{x}=-\sin \left(\theta_{1}+\theta_{2}+\theta_{3}+\theta_{4}+\theta_{5}\right) \\
a_{x}=0
\end{gathered}
$$

$p_{x}=l_{1} \cos \left(\theta_{1}\right)+l_{2} \cos \left(\theta_{1}+\theta_{2}\right)+$

$$
\begin{gathered}
l_{3} \cos \left(\theta_{1}+\theta_{2}+\theta_{3}\right)+l_{4} \cos \left(\theta_{1}+\theta_{2}+\theta_{3}+\theta_{4}\right)+ \\
l_{5} \cos \left(\theta_{1}+\theta_{2}+\theta_{3}+\theta_{4}+\theta_{5}\right) \\
n_{y}=\sin \left(\theta_{1}+\theta_{2}+\theta_{3}+\theta_{4}+\theta_{5}\right) \\
o_{y}=\cos \left(\theta_{1}+\theta_{2}+\theta_{3}+\theta_{4}+\theta_{5}\right) \\
a_{y}=0
\end{gathered}
$$

$$
p_{y}=l_{1} \sin \left(\theta_{1}\right)+l_{2} \sin \left(\theta_{1}+\theta_{2}\right)+
$$$$
l_{3} \sin \left(\theta_{1}+\theta_{2}+\theta_{3}\right)+l_{4} \sin \left(\theta_{1}+\theta_{2}+\theta_{3}+\theta_{4}\right)+
$$$$
l_{5} \sin \left(\theta_{1}+\theta_{2}+\theta_{3}+\theta_{4}+\theta_{5}\right)
$$

$$
n_{z}=0 ; \quad o_{z}=0 ; \quad a_{z}=0 ; \quad p_{z}=0
$$

Las variables $\theta_{1}, \theta_{2}, \theta_{3}, \theta_{4}$ y $\theta_{5}$ corresponden a los ángulos de cada articulación y $l_{1}, l_{2}, l_{3}, l_{4}$ y $l_{5}$ a la longitud de cada eslabón. Para dar solución a la cinemática inversa, a pesar de los diferentes métodos que existen en la actualidad [16], se utilizó los métodos geométricos debido a su bajo consumo computacional. Para esto se dividió el área de trabajo del pez robótico en 4 subáreas que se encuentran entre un radio máximo $r_{\text {Tmax }}$, correspondiente al radio cuando todos los eslabones están paralelos entre sí, y un radio mínimo $r_{T \min }$, que se presenta cuando todos los eslabones han alcanzado su rango máximo, como se presenta en la Figura 6. Los valores obtenidos para $r_{\text {Tmax }}$ y $r_{\text {Tmin }}$ fueron $51,1 \mathrm{~cm}$ y $36,754 \mathrm{~cm}$, respectivamente.

(a)

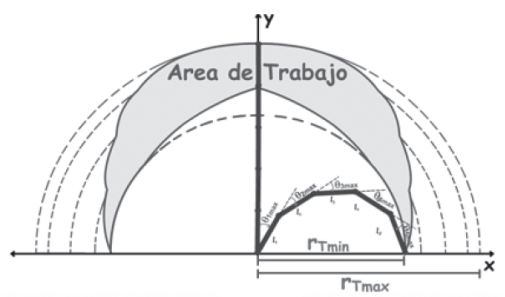

(b)

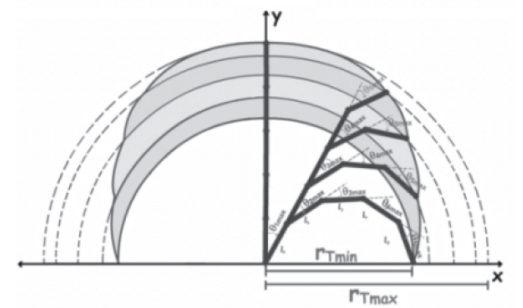

(c)

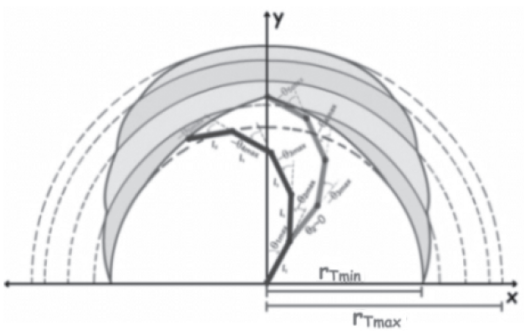

Figura 6. (a) Representación del área de trabajo del pez robótico, (b) subáreas de trabajo para cada articulación del pez robótico, (c) límite inferior del área de trabajo.

Para implementar el diseño virtual se dividió la estructura en 3 partes. La cabeza, en donde se ubica la circuitería de control y alimentación de la plataforma; el cuerpo, en donde se encuentran las cinco articulaciones del robot, cada una representada por una base. En cada articulación se ubicará un servomotor acoplado a un sistema de engranaje que 
funcionará como actuador y, por último, la cola del pez robótico, como se presenta en la Figura 7.

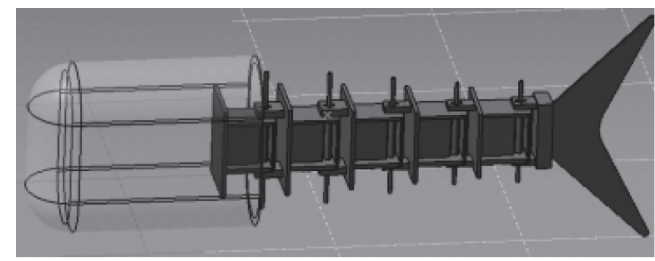

(a)

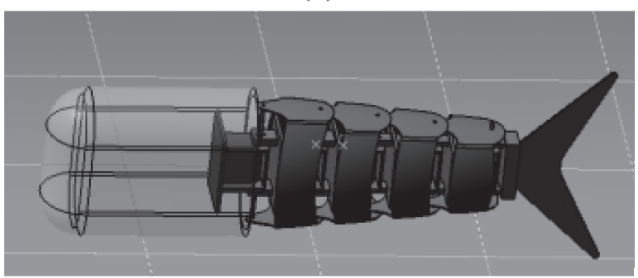

(b)

Figura 7. a) Esqueleto del pez robótico, b) cubierta del pez robótico.

Las longitudes y las masas para cada eslabón, incluyendo la cubierta, se presentan a continuación en la Tabla 1. Para cada eslabón el material seleccionado fue el aluminio AL7075.

Tabla 1. Parámetros de longitud y masa del pez robótico para los 5 grados de libertad.

\begin{tabular}{|c|c|c|}
\hline ESLABÓN & $\begin{array}{c}\text { LONGITUD } \\
{[\mathbf{c m}]}\end{array}$ & $\begin{array}{c}\text { MASA } \\
{[\mathbf{k g}]}\end{array}$ \\
\hline 1 & 8,4 & 0,3110 \\
\hline 2 & 8,4 & 0,2950 \\
\hline 3 & 8,4 & 0,2650 \\
\hline 4 & 8,4 & 0,2500 \\
\hline 5 & 17,5 & 0,3160 \\
\hline
\end{tabular}

\section{ALGORITMO DE CONTROL}

Para el algoritmo de control se consideró el pez robótico como un robot móvil, es decir, el objetivo es descubrir una trayectoria a lo largo de un marco global descrito por un rectángulo con área 600x500 $\mathrm{cm}^{2}$.

\section{Control de Trayectoria}

Para el control de trayectoria se deben considerar 3 ángulos: $\theta_{\text {cabeceo }}, \theta_{\text {obj }}$ y $\theta_{\text {error }}$. El $\theta_{\text {cabeceo }}$ corresponde al ángulo de cabeceo u orientación del pez robótico respecto de un eje de referencia, en este caso, el eje $\mathrm{x} ; \theta_{o b j}$ representa el ángulo formado entre el punto objetivo y el eje de referencia, y $\theta_{\text {error }}$, corresponde a la diferencia entre el ángulo del objetivo y el ángulo de cabeceo del robot [15], como en la Figura 8.

Se utilizó la lógica difusa [10, 18, 23, 28, 29] para el control de trayectoria por ser un algoritmo que suministra una metodología efectiva y eficiente para el desarrollo de controladores no lineales sin la necesidad de usar cálculos matemáticos complejos. Para esto se consideró que el ángulo de cabeceo del pez $\theta_{\text {cabeceo }}$ oscilaba entre dos rangos: $\left[0^{\circ} 180^{\circ}\right]$, y $\left[0^{\circ}-180^{\circ}\right]$.

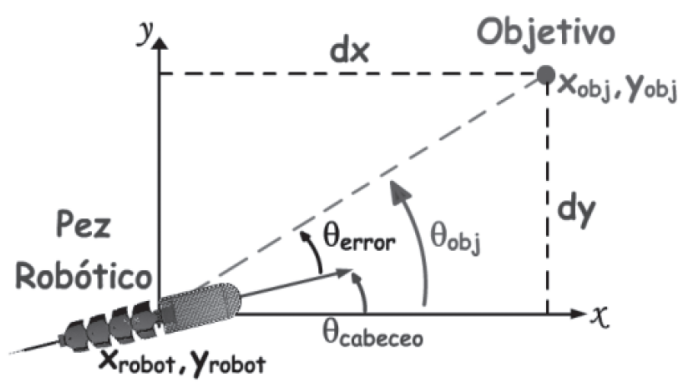

Figura 8. Control de orientación del pez robótico.

El ángulo $\theta_{\text {error }}$ puede ser positivo o negativo. $\mathrm{Si}$ el pez robótico gira en torno a las manecillas del reloj se considera positivo. Por otro lado, si el pez gira en sentido contrario a las manecillas se considera negativo. El ángulo de cabeceo $\theta_{\text {cabeceo }} \mathrm{y}$ el ángulo del objetivo $\theta_{o b j}$ oscilan en un rango entre $\left[0^{\circ}-180^{\circ}\right]$ y $\left[0^{\circ}-180^{\circ}\right]$. El diagrama para el control de orientación es presentado en la Figura 9, el que consta de dos entradas: una para el ángulo objetivo $\theta_{o b j}$ y la otra para el ángulo de posición inicial del pez robótico $\theta_{\text {inicial }}$. Estas señales de entrada pasan por un bloque de corrección de entradas, el que se encarga de posicionar todos los ángulos respecto de un mismo punto de referencia. Las salidas de este bloque son llevadas al fusificador, en donde los valores numéricos son convertidos en valores difusos para poder ser procesados por el mecanismo de inferencia [28]. El mecanismo de inferencia toma los valores generados por el fusificador y los procesa considerando la base de reglas para generar la salida del sistema difuso. La salida de este sistema se lleva a un defusificador, el que convierte los valores difusos en valores numéricos. El valor obtenido en este bloque, corresponde al ángulo $\theta_{c a b}$ indicando cuánto debe girar el pez. Por último, este valor del ángulo de cabeceo obtenido debe pasar a un bloque al que se le denominó Corrección Salidas, con el fin de 
entregar el valor real de giro respecto del punto de referencia del pez robótico.

Las funciones de pertenencia seleccionadas para las entradas y salidas del controlador difuso fueron tipo triangular.

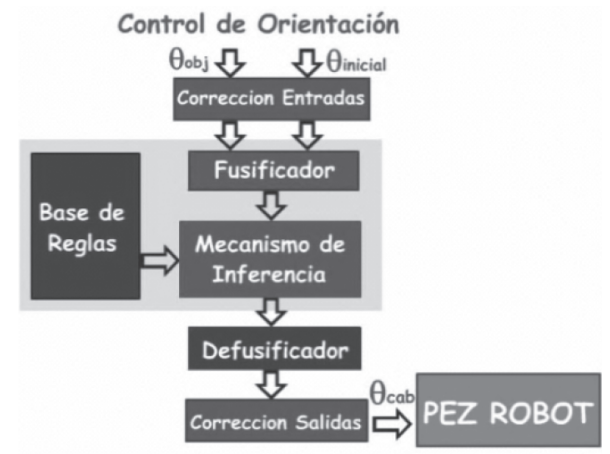

Figura 9. Diagrama del controlador difuso para el control de orientación del pez robótico.

Se seleccionó este tipo de función de pertenencia por dos razones: una de ellas es porque conlleva a un gasto computacional mucho menor respecto de otras funciones de pertenencia [15], y la otra, por su simplicidad en la implementación. Para las entradas del controlador se consideró el área de trabajo en un plano cartesiano con 4 cuadrantes, en

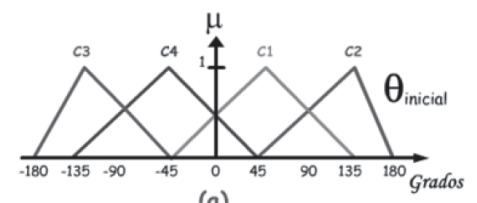

(a)

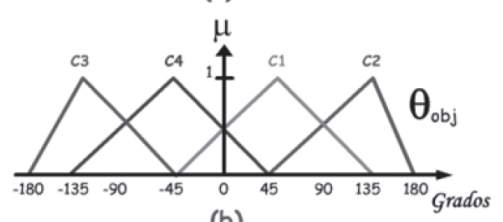

(b)

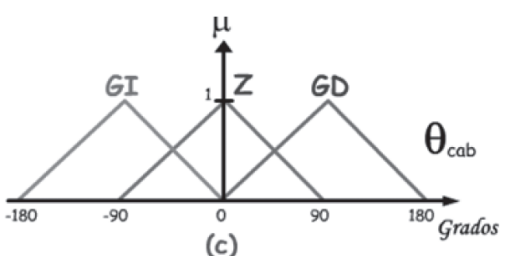

(c)

Figura 10. Funciones de Pertenencia de las variables de entrada y salida para el control de orientación. a) Ángulo Inicial del Pez Robótico $\theta_{i n i}$, b) Ángulo del Objetivo $\theta_{o b j}$, c) Ángulo de Cabeceo $\theta_{c a b}$. donde el cuadrante 1 corresponde a ángulos entre 0 y 90 grados, el cuadrante 2 a ángulos entre 90 y 180 grados, el cuadrante $3 \mathrm{a}$ ángulos entre $-180 \mathrm{y}$ -90 grados y el cuadrante 4 entre -90 y 0 grados. Para las salidas se utilizó este mismo criterio. Las funciones de pertenencia se presentan en la Figura 10 y las reglas en la Tabla 2.

Tabla 2. Reglas para el controlador difuso de control de orientación. Z=cero, GD=Girar Derecho, $\mathrm{GI}=$ Girar Izquierda, $\mathrm{C} 1=$ Cuadrante 1, C2 $=$ Cuadrante $2, \mathrm{C} 3=$ Cuadrante 3 , C4=Cuadrante 4 .

\begin{tabular}{|c|c|c|c|c|}
\hline & C1 & C2 & C3 & C4 \\
\hline C1 & Z & GD & Z & GI \\
\hline C2 & GI & Z & GD & Z \\
\hline C3 & Z & GI & Z & GD \\
\hline C4 & GD & Z & GI & Z \\
\hline
\end{tabular}

\section{Control de velocidad}

Para el algoritmo de control de velocidad se debe considerar 3 distancias, la primera corresponde a la distancia en la que el pez robótico está acelerando hasta que este alcance su máxima velocidad, y a partir de esta su velocidad se mantiene constante [15]. La segunda corresponde a la distancia en la que el pez robótico debe empezar a desacelerar hasta que su velocidad alcance un valor cercano a cero, y la tercera corresponde a la distancia a la que el pez robótico debe detenerse, como se presenta en la Figura 11 .

En la distancia $d_{l}$ el pez ha acelerado desde cero hasta alcanzar la máxima velocidad. Si la distancia del pez robótico y el objetivo está entre $d_{1}$ y $d_{2}$, este conserva una velocidad constante correspondiente a la velocidad máxima, y si la distancia es mayor a $d_{2}$, el pez robótico debe empezar a desacelerar. En la Figura 12 y la ecuación (11) se presenta el perfil de velocidad del pez robótico.

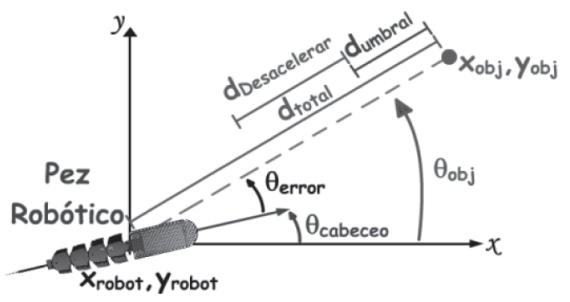

Figura 11. Control de velocidad del pez robótico. 


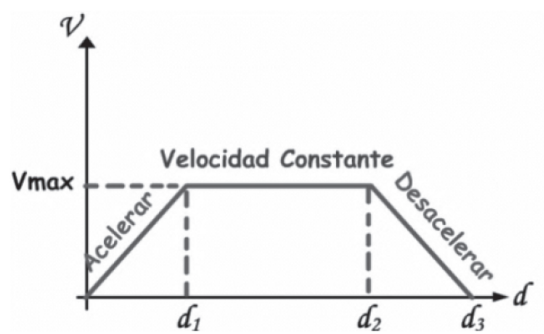

Figura 12. Perfil de velocidad del pez robótico.

$$
\begin{aligned}
& V_{p e z}= \\
& \left\{\begin{array}{cl}
\frac{V_{\max }}{2}\left(1-\cos \left(\frac{\pi d}{d_{1}}\right)\right. & ; 0 \leq d<d_{1} \\
V_{\max } & ; d_{1} \leq d<d_{2} \\
\frac{V_{\max }}{2}\left(1-\cos \left(\frac{\pi\left[d-\left(d_{2}+d_{3}\right)\right]}{d 2}\right)\right. & ; d_{1} \leq d<d_{2}+d_{3} \\
0 & ; d_{3} \leq d
\end{array}\right\}
\end{aligned}
$$

\section{ANÁLISIS Y RESULTADOS}

Para esta sección, los resultados se han presentado en dos partes. En la primera, el pez robótico es considerado como un robot estático, es decir, el objetivo es posicionar la cola del pez en un punto determinado dentro de su área de trabajo, pero sin que se desplace. En la segunda, el pez es considerado como un robot móvil, cuyo objetivo es describir una trayectoria determinada dentro de un rectángulo. Para la primer parte los resultados presentados se centran en la cinemática directa e inversa, la posición, velocidad, aceleración y fuerzas en cada uno de los eslabones del cuerpo del pez robótico. En la segunda parte los resultados se centran en el algoritmo de control.

En la Tabla 3 se presentan los errores absolutos para cada ángulo. El máximo error presentado corresponde al del punto 3 , con un valor de 0,44 grados, indicando que la máxima diferencia entre el ángulo obtenido de la cinemática directa y el obtenido de la cinemática inversa es de 0,44 grados, un valor relativamente pequeño en este caso.

Las posiciones, velocidades y aceleraciones angulares para cada uno de los eslabones en grados, grados/ segundos y grados/segundos ${ }^{2}$, respectivamente, se presentan en la Tabla 3. La máxima posición que puede tomar cada uno de los eslabones es 30 grados.
Tabla 3. Resultados de los errores absolutos para cada ángulo al comparar los resultados obtenidos de la cinemática inversa con la cinemática directa.

\begin{tabular}{|c|c|c|c|c|c|c|c|}
\hline & $\mathbf{X}$ & $\mathbf{Y}$ & \multicolumn{5}{|c|}{ Error } \\
& & & & \multicolumn{1}{|c|}{$\theta_{C D}-\theta_{c l} l$} & \\
\hline & & & & & & & \\
\hline $\mathbf{1}$ & $-8,75$ & 48,8 & 0,13 & 0 & 0 & 0 & 0,4 \\
\hline $\mathbf{2}$ & $-33,9$ & 14,3 & 0,25 & 0,3 & 0 & 0 & 0 \\
\hline $\mathbf{3}$ & $-26,7$ & 30,2 & 0,37 & 0,44 & 0 & 0 & 0 \\
\hline $\mathbf{4}$ & 36,3 & 17,9 & 0 & 0,1 & 0 & 0 & 0 \\
\hline $\mathbf{5}$ & 0,91 & 45,5 & 0,2 & 0 & 0,27 & 0 & 0 \\
\hline $\mathbf{6}$ & 38 & 6,93 & 0,3 & 0,2 & 0 & 0 & 0 \\
\hline $\mathbf{7}$ & $-16,8$ & 46,6 & 0,06 & 0 & 0 & 0 & 0 \\
\hline $\mathbf{8}$ & $-21,9$ & 40,9 & 0 & 0 & 0 & 0 & 0 \\
\hline $\mathbf{9}$ & $-12,1$ & 48,8 & 0,07 & 0 & 0 & 0 & 0,1 \\
\hline $\mathbf{1 0}$ & 34 & 32,7 & 0 & 0 & 0 & 0 & 0 \\
\hline
\end{tabular}

La máxima velocidad alcanzada se encuentra en la cola del pez robótico que corresponde a 56,2 grados/segundos y la máxima aceleración es de 4,33 grados/segundos ${ }^{2}$, como se presenta en la Figura 13. Para los cálculos de las posiciones, velocidades y aceleraciones de cada articulación se utilizaron los parámetros mostrados en la Tabla 1 y para obtener las respuesta de posición, velocidad, aceleración, trayectoria y fuerza del pez robótico se utilizaron las técnicas nombradas anteriormente en el artículo y el Robotic Toolbox de Peter Corke con los algoritmos

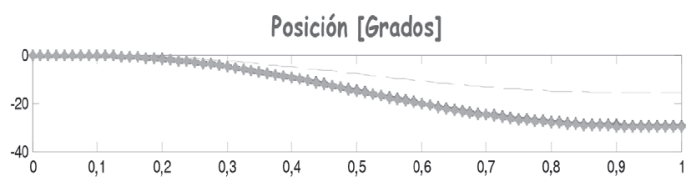

Velocidad Angular [Grados/s]

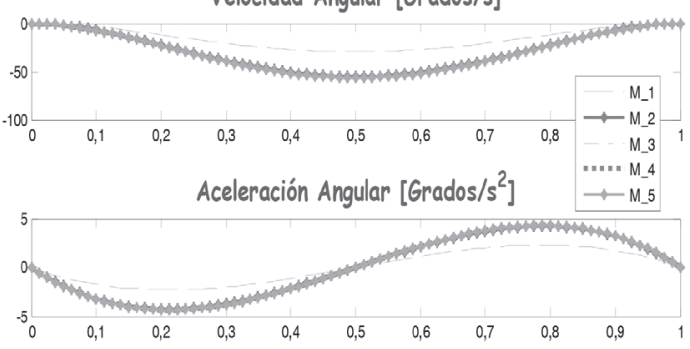

Figura 13. Posiciones, velocidades y aceleraciones angulares de cada uno de los eslabones del pez robótico. M_1=Motor de la articulación 1, M_2=Motor 2, M_3=Motor 3, M_4= Motor 4, M_5=Motor 5 . 
propuestos en [26]. En los resultados presentados no se consideraron fuerzas de arrastre ni la fricción que genera con el agua.

Las posiciones $x, y$ y $z$ de la cola del robot y la trayectoria generada se presentan en la Figura 14. Debido a que se limitó el movimiento del pez solo a movimientos en el plano xy, es decir, no se consideró el movimiento de cabeceo, la posición en el eje z debe ser constante, en este caso se consideró un offset de 1, como se observa en la parte superior de la Figura 14. Además, porque se consideró que el pez robótico va desde su posición de reposo (posición en la que todas sus articulaciones se encuentran alineadas) hasta alcanzar su máximo giro, la trayectoria que involucra este movimiento debe conservar una forma elíptica, como se presenta en la parte inferior de la Figura 14.

Las fuerzas inerciales, coriolis, centrífugas y de gravedad en el pez robótico cuando va desde su
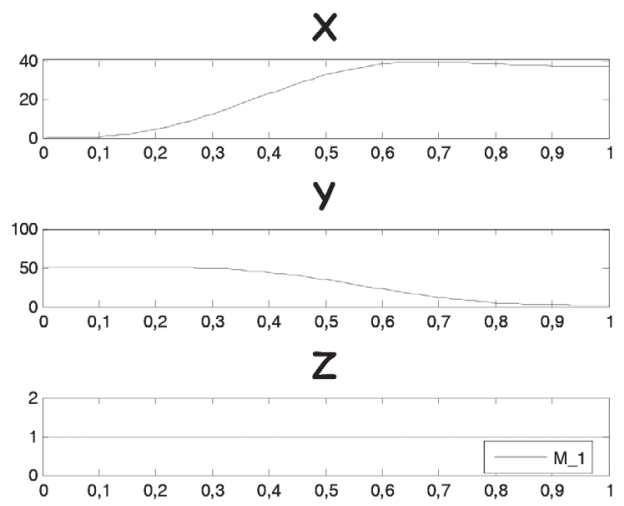

TRAYECTORIA

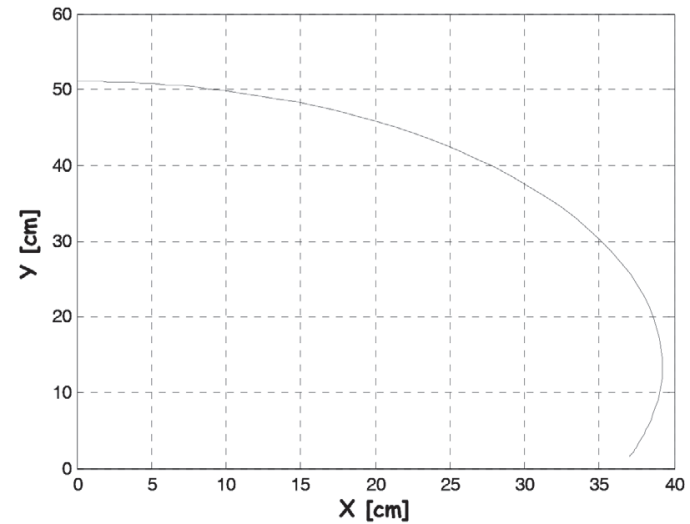

Figura 14. Posición cartesiana y trayectoria generada por el pez robótico. posición inicial o reposo, es decir, cuando todos los eslabones están alineados, hasta su posición final, se presentan en la Figura 15. La fuerza que más influye en el movimiento es la fuerza inercial. Ya que no hay cambio en la profundidad del pez robótico, y tampoco se considera el movimiento de cabeceo, el efecto de la fuerza de la gravedad es nulo.

El torque máximo en el pez robótico se presenta en la primera articulación, ya que es la que sostiene todas las demás articulaciones del pez robótico. El valor máximo alcanzado por esta articulación fue de 21,6 kgf-cm, como se presenta en la Figura 16.

Considerando ahora el pez robótico como un robot móvil, el objetivo se centra en ir de un punto de partida a un punto final. Para esto, el pez robótico debe primero orientarse hacia su punto objetivo y una vez que esté orientado empezará a desplazarse

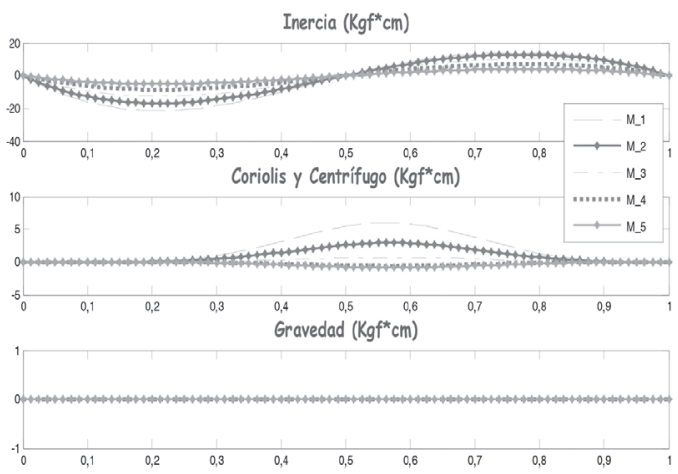

Figura 15. Fuerzas de inercia, coriolis y centrífuga y gravedad que se presentan en el pez robótico.

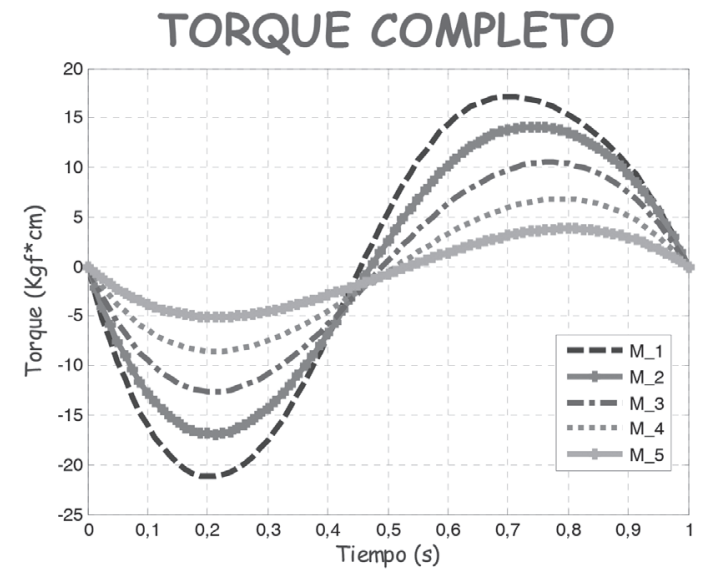

Figura 16. Torque que se presenta en el pez robótico. 
de manera lineal hasta alcanzarlo. Si el objetivo es un punto detrás del pez robótico, este tendrá que girar hasta que todas sus articulaciones alcancen sus máximos ángulos de giro, y dependiendo de la ubicación de dicho punto, este girará hacia la derecha o izquierda.

En el algoritmo diseñado e implementado con lógica difusa se consideró que si el punto objetivo se ubicaba entre 0 y 180 grados, tomando como origen la cabeza del pez robótico, este giraba en sentido contrario de las manecillas del reloj, y en cualquier otro caso giraba en el sentido de las manecillas del reloj. En la Figura 17 el objetivo se ubica a -170 grados del pez robótico y se presenta el giro del pez robótico en sentido de las manecillas del reloj.

Cuando la orientación es alcanzada, los ángulos vuelven a su posición inicial llevando todos los ángulos de los eslabones del robot a cero para empezar a desplazarse hacia el punto. Cuando se ha alcanzado determinada distancia, el pez disminuye su velocidad y cuando se alcanza la distancia umbral entre el objetivo y el pez robótico, el robot se detiene.

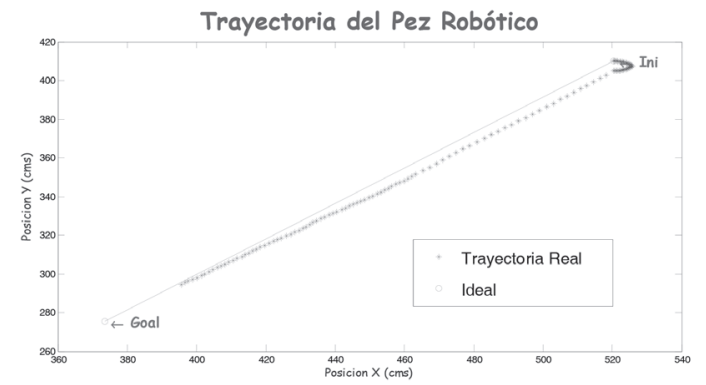

Figura 17. Trayectoria realizada por el pez robot en busca del objetivo.

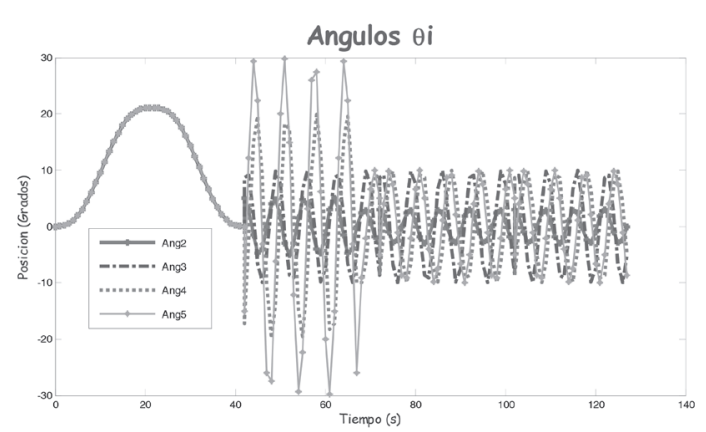

Figura 18. Posición angular de cada una de los eslabones del pez robótico para alcanzar el objetivo.
En la Figura 18 se observa que al inicio la amplitud de las posiciones angulares de todas las articulaciones se incrementan hasta alcanzar su máxima amplitud y después vuelven a su punto de partida. Esta primera etapa corresponde a la fase en la que el pez robótico gira para ubicarse en dirección del objetivo. Luego, cada una de las articulaciones se mueven con diferentes amplitudes, a una determinada velocidad y, por último, las amplitudes de estas articulaciones decrecen, indicando que la distancia mínima o umbral entre el objetivo y el pez robótico ha sido alcanzada, y por lo tanto debe disminuir su velocidad. Los perfiles de las velocidades y aceleraciones angulares de cada articulación son presentadas en las Figuras 19 y 20, respectivamente.

La amplitud de las ondas sinusoidales representan los ángulos máximos alcanzados por cada una de las articulaciones del pez robótico. Así, cuando el pez robótico gira hacia la derecha, todas las articulaciones se desplazan hacia los 30 grados positivos como se presentó al inicio de la Figura 6.

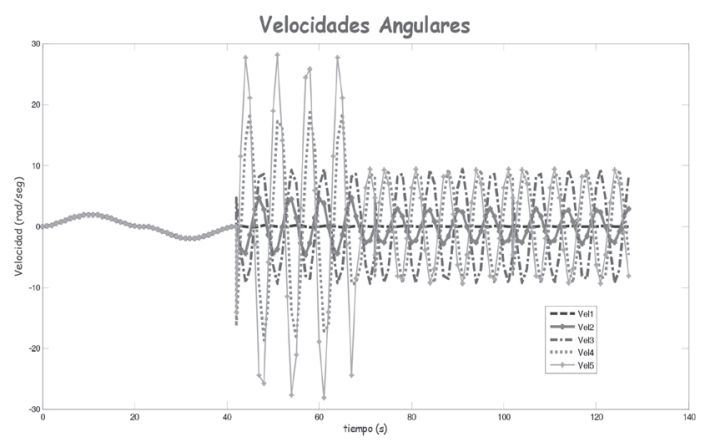

Figura 19. Velocidad angular de cada una de los eslabones del pez robótico para alcanzar el objetivo.

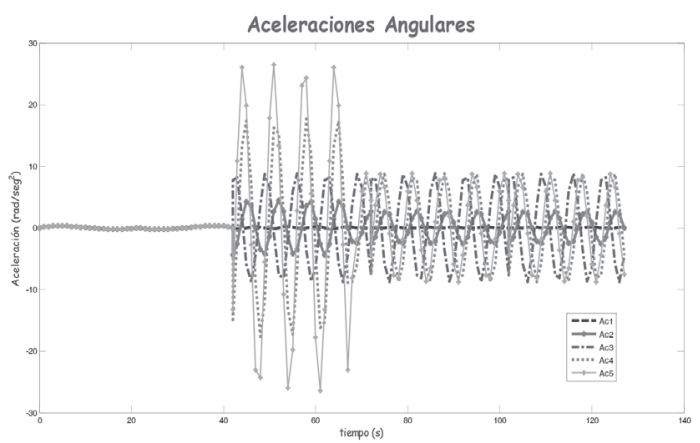

Figura 20. Aceleración angular de cada una de los eslabones del pez robótico para alcanzar el objetivo. 
La amplitud de la onda seno depende del radio de giro del pez, es decir, cuanto mayor sea el radio de giro del pez, mayor será la amplitud de las ondas seno de cada articulación. Cuando el pez robótico está navegando hacia adelante, las ondas seno oscilan entre un valor de \pm 30 grados, dependiendo de la velocidad y la distancia del objetivo.

\section{CONCLUSIONES}

En el desarrollo del modelo del pez robótico se seleccionó solo uno de los modos de nado de los movimientos (BCF) presentados: el modo subcarangiforme. Sin embargo, debido a los cinco grados de libertad seleccionados para el cuerpo, el tipo de análisis desarrollado tanto para la cinemática como para la parte estructural del pez robótico puede ser aplicado para los otros modos BCF, ya que se conserva una relación entre los movimientos oscilatorios y ondulatorios, e incluso, puede ser expandido a los modos de los peces que usan sus aletas pares e impares (MPF), considerando que esta cinemática ya no sería aplicada a sus cuerpos sino a cada una de aletas.

En simulación se observó que el modelo de la cinemática inversa desarrollado presentó un buen comportamiento al momento de ser comparada con la cinemática directa, con un error máximo de $1,47 \%$, un error que para este proyecto es considerado aceptable. Esta estimación del error se obtiene siempre que se tengan presentes las consideraciones realizadas, ya que este modelo de robot a pesar de ser catalogado como un robot planar redundante, no todos los movimientos obtenidos en estos tipos de robots se lograron en el modelo desarrollado.

Para el análisis de fuerzas se concluyó mediante simulación que el mayor torque se presenta cuando el pez robótico gira, ya que todas sus articulaciones se ven forzadas a alcanzar su límite. La velocidad angular de cada articulación es un factor muy importante para el efecto de torques. Cuanto mayor sea la velocidad angular, menor es el torque que se necesita para mover cada eslabón del robot, y como era de esperarse, el mayor torque se presentó en el primer eslabón.

Por ser un robot que presenta un modelo no lineal y su análisis es complejo, incluyendo las leyes de control, se optó por realizar un control de trayectoria usando la lógica difusa, presentando un buen comportamiento de acuerdo con los resultados obtenidos para el control de trayectoria.

Este proyecto fue desarrollado en su totalidad considerando los conceptos teóricos que rigen el comportamiento de los peces robóticos, y aunque no se realizó una validación física, corresponde a un gran avance en este tipo de robots a nivel subacuático, ya que presenta la base para el desarrollo de una plataforma subacuática bioinspirada a nivel físico.

\section{AGRADECIMIENTOS}

Esta investigación fue realizada con el apoyo de los grupos de investigación de las Escuelas de Ingeniería Eléctrica, Electrónica y Mecánica de la Universidad del Valle, Cali, Colombia.

\section{REFERENCIAS}

[1] F.E. Fish and D.M. Kocak. "Biomimetics and Marine Technology". Marine Technology Society Journal: The International, Interdisciplinary Society Devoted to Ocean and Marine Engineering, Science, and Policy. Vol. 45, Issue 4, pp. 10-236. July/August, 2011. ISSN: 0025-3324.

[2] K.H. Low. "Modelling and parametric study of modular undulating fin rays for fish robots". Mechanism and Machine Theory. Vol. 44, Issue 3, pp. 615-632. March, 2009. ISSN: 0094-114X. DOI: 10.1016/j.mechmach theory.2008.11.009.

[3] M. Sfakiotakis, D.M. Lane and J.B.C. Davies. "Review of Fish Swimming Modes for Aquatic Locomotion". IEEE Journal of Oceanic Engineering. Vol. 24, Issue 2, pp. 237-252. April, 1999. ISSN: 0364-9059. DOI: $10.1109 / 48.757275$.

[4] S. Hirose, S. Sugita, K. Ogami, G. Michele and K. Takita. "A Study on the Mechanism and Locomotion Strategy for New SnakeLike Robot Active Cord Mechanism-Slime model 1 ACM-S1". Journal of Robotics and Mechatronics. Vol. 20, Issue 2, pp. 302-310. December, 2008. ISSN: 0915-3942 (Print) / 1883-8049 (Online). URL: http://www. fujipress.jp/JRM/ 
[5] R.W. Blake. "The mechanics of labriform motion I. Labriform locomotion in the angelfish (pterophyllum eimekei): An analysis of the power stroke". Journal of Experimental Biology. Vol. 82, pp. 255-271. September, 1979. ISSN: 0022-0949 (Print) / 1477-9145 (Online). URL: http://jeb.biologists.org/

[6] E.G.Drucker and G.V.Lauder. "A Hydrodinamic Analysis of Fish Swimming Speed: Wake Structure and Locomotor Force in Slow and Fast Labriform Swimmers". The Journal of Experimental Biology. Vol. 203, Issue 16, pp. 2379-2393. August, 2000. ISSN: 00220949 (Print) / 1477-9145 (Online). URL: http://jeb.biologists.org/

[7] J. Palmisano, R. Ramamurti, K.-J. Lu, J. Cohen, W. Sandberg and B. Ratna. "Design of a biomimetic controlled curvature robotic pectoral fin". 2007 IEEE International Conference on Robotics and Automation. Roma, Italy. April 10-14, 2007.

[8] W. Wang, J. Yu, M. Wang and R. Ding. "Mechanical Design and Preliminary Realization of Robotic Fish with multiple Control Surfaces". 29th Chinese Control Conference. Beijing, China. July 29-31, 2010.

[9] J. Yu, M. Wang, W. Wang, M. Tan and J. Zhang. "Design and Control of a Fish-Inspired Multimodal Swimming Robot”. 2011 IEEE International Conference on Robotics and Automation. Shanghai, China. May 9-13, 2011.

[10] J. Liu, H. Hu, and D. Gu. "A Hybrid Control Architecture for Autonomous Robotic Fish". International Conference on Intelligent Robots and Systems. Beijing, China. October 9-15, 2006.

[11] G.S. Triantafyllou, M.S. Tryantafyllou and M.A. Grosenbaugh. "Optimal Thrust development in oscillating foils with application to fish propulsion". Journal of Fluids Structures. Vol. 7, Issue 2. pp. 205224. February, 1993. ISSN: 0889-9746. DOI: 10.1006/jfls.1993.1012.

[12] M.J. Lighthill. "Hydromechanics of aquatic animal propulsion". Journal of Marine Science and Application. Vol. 10, Issue 2, pp. 163-174. June, 2011. ISSN: 1671-9433 (Print) / 1993-5048 (Online). URL: http://link. springer.com/journal/11804 DOI: $10.1007 /$ s11804-011-1056-3.
[13] W.S. Vorus and B.M. Taravella "Anguilliform fish propulsion of highest hydrodynamic efficiency". Annual Review of Fluid Mechanics. Vol. 1, Issue 1, pp. 413-466. January, 1969. DOI: 10.1146/annurev. fl.01.010169.002213.

[14] M.S. Triantafyllou and G.S. Tryantafyllou. "Los Robots de Natación Autónoma". Investigación y Ciencia. $\mathrm{N}^{\circ} 224$, pp. 26-32. Mayo, 1995. ISSN: 0210-136X.

[15] J. Yu, M. Tan, S. Wang and E. Chen. "Development of a Biomimetic Robotic Fish and Its Control Algorithm". IEEE Transactions on Systems: Man and Cybernetics Part B: Cybernetics. Vol. 34, Issue 4, pp. 1798-1810. August, 2004. ISSN: 1083-4419. DOI: 10.1109/TSMCB. 2004.831151.

[16] D. Tolani, A. Goswami and N.I. Badler. "Real-Time Inverse Kinematics Techniques for Anthropomorphic Limbs". Graph Models. Vol. 62, Issue 5, pp. 353-388. May, 2000. ISSN: 1524-0703. DOI: $10.1006 /$ gmod.2000.0528.

[17] K.A. Morgansen, V. Duindam, R.J. Mason, J.W. Burdick and R.M. Murray. "Nonlinear Control Methods for Planar Carangiform Robot Fish Locomotion". IEEE International Conference on Robotics and Automation. Vol. 1, pp. 427-434. April, 2006. ISBN: 0-7803-6576-3. DOI: 10.1109/ ROBOT.2001.932588.

[18] J.D. Liu and H. Hu. "Biologically Inspired Behaviour Design for Autonomous Robotic Fish". International Journal of Automation and Computing. Vol. 3, Issue 4, pp. 336-347. October, 2006. ISSN: 1476-8186 (Print) / 1751-8520 (Online). URL: http://link. springer.com/journal/11633. DOI: 10.1007/ s11633-006-0336-x.

[19] C.F. Alba Ayala, R.C. Mossos and D.R. Mancera Guevara. "Plataforma robot subacuática propulsada por aletas pectorales". Ingeniería y Universidad. Vol. $9 \mathrm{~N}^{\circ} 1$, p. 84. Junio 2005. ISSN: 0123-2126.

[20] U. Scacco, G. la Mesa and M. Vacchi. "Body morphometrics, swimming diversity and niche in demersal sharks: a comparative case study from the Mediterranean Sea". Scientia Marina. Vol. 74, Issue 1, pp. 37-53. March, 2010. ISSN: 0214-8358. DOI: 10.3989/ scimar.2010.74n1037. 
[21] J. González-Gómez and E. Boemo. "Motion of Minimal Configurations of a Modular Robot: Sinusoidal, Lateral Rolling and Lateral shift". International Conference on Climbing and Walking Robots, pp. 667-674. Springer Berlin. Heidelberg, Germany. September, 2005. ISBN: 978-3-540-26413-2.

[22] G.V. Lauder and P.G.A Madden. "Fish Locomotion: Kinematics and Hydrodynamics of flexible foil-like fins". Experiments in Fluids. Vol. 43, Issue 5, pp. 641-653. November, 2007. ISSN: 0723-4864 (Print) / 1432-1114 (Online). URL: http://link. springer.com/journal/348. DOI: $10.1007 /$ s00348-007-0357-4.

[23] Y. Hao. "Fuzzy Control and Modeling, Analytical Foundations and Applications". $1^{\circ}$ Edition. Wiley-IEEE Press, pp. 1-304. New York, USA. ISBN-10: 0780334973, ISBN-13：978-0780334977. DOI: 10.1109/9780470544730. Copyright 2000.

[24] J. González-Gómez, H. Zhang and E. Boemo. "Bioinspiration and Robotics: Walking and Climbing Robots". Universidad Autónoma de Madrid. Advanced Robotic Systems Interantional and I-Tech Education and Publishing, pp. 403-428. Vienna, Austria. ISBN: 978-3-902613-15-8. Year 2007.

[25] C.C. Lindsey. "Fish Physiology". First edition. Academic Press. Vol. 7, pp. 1-100. London,
United Kingdom. ISBN: 0-12-350407-4. Copyright 1978.

[26] P.I. Corke. "Robotics, Vision and Control: Fundamental Algorithms in Matlab". Fourth edition. Springer. Vol. 14, pp 1-218. ISBN: 978-3-642-20143-1. Copyright Peter Corke 2012.

[27] H. Koichi and S. Kawai. "Hydrodinamic Performance of Stream-Lined Body". National Maritime Research Institute. Date of visit: July 20, 2011. URL: http://www.nmri. go.jp/eng/khirata/fish/experiment/upf2001/ body_e.html

[28] J.A. López. "Lógica Difusa Introducción y Conceptos Básicos". Curso Online de Inteligencia Computacional. Fecha de consulta: 15 de noviembre de 2011. URL: http://members.tripod.com/jesus_alfonso_ lopez/FuzzyIntro2.html

[29] G.M. Luna. "Introducción a la Lógica Difusa". Centro de Investigación y Estudios Avanzados del IPN. Fecha de consulta: 18 de noviembre de 2011. URL: http://delta. cs.cinvestav.mx/ gmorales/ldifll/ldifll.html

[30] A. Barriento, L.F. Peñin, C. Balaguer and R. Aracil. "Fundamentos de Robótica". $2^{\mathrm{a}}$ Edición. McGraw-Hill. Vol. 1, pp. 1-327. ISBN: 9788448156367. Madrid, España. Derechos Reservados 2007. 